\title{
CLCN2 chloride channel mutations in primary aldosteronism
}

\section{C4 \\ Our findings \\ will hopefully \\ allow \\ physicians \\ to make a \\ diagnosis in \\ more patients}

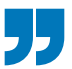

Primary aldosteronism is an important cause of hypertension. A number of mutations that encode cation channels and ATPases that regulate calcium influx and alter the downstream production of aldosterone synthase in glomerulosa cells have been identified, but the pathogenesis of some cases remains unknown. Two new studies now demonstrate that gain-of-function mutations in CLCN2, which encodes the chloride channel $\mathrm{ClC} 2$, increase chloride conductance at resting potentials, producing membrane depolarization of glomerulosa cells and inducing expression of aldosterone synthase, the key enzyme involved in aldosterone production. "This is the first time that a chloride channel has been linked to the regulation of aldosterone production from the adrenal

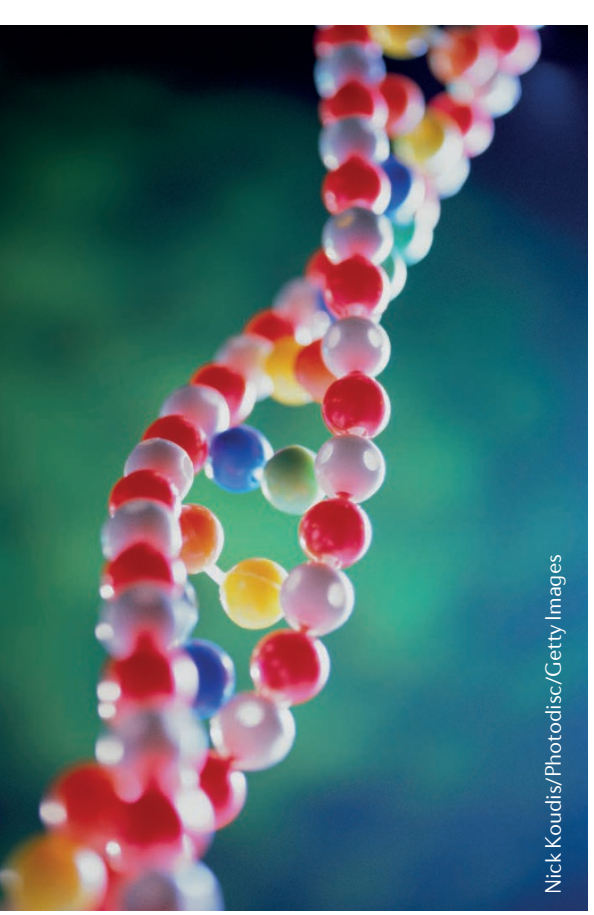
cortex," explains Fabio Fernandes-Rosa, author of one of the studies. "In our study, 17 individuals among eight families had CLCN2 mutations; several apparently unrelated families carried the exact same mutation and two mutations were de novo, so the genetic evidence that this is the underlying disease gene is overwhelming," adds Ute Scholl, an author on the second study. "Our findings will hopefully allow physicians to make a diagnosis in more patients and families with the disease."

Scholl and colleagues initiated their study to investigate the genetic basis of primary aldosteronism in a large family first described $>25$ years ago. In collaboration with Michael Stowasser and Richard Gordon, they performed exome sequencing in three affected individuals, leading to the identification of a mutation in CLCN2. Further collaboration with Richard Lifton led to the identification of seven additional families with CLCN2 mutations. The researchers then used immunohistochemistry to confirm expression of $\mathrm{ClC} 2$ in human adrenal zona glomerulosa, consistent with a role for this channel in regulating aldosterone production. Whole-cell patch-clamp electrophysiology of HEK293 cells transfected with wild-type or mutated $\mathrm{ClC} 2$ showed that the mutations activated the channel, allowing more chloride to leave the cell and inducing a greater change in membrane potential than the wild-type channel. Transfection of mutant channels into human H295R adrenocortical cancer cells - a model for studying the production of aldosterone - demonstrated that the depolarizing change in membrane potential was associated with the increased production of CYP11B2, which encodes aldosterone synthase. "The identification of mutations in $\mathrm{ClC} 2$ solves a conundrum that had stumped researchers for many years," explains Scholl. "Interestingly, although CLCN2 encodes a chloride channel that is highly expressed in aldosterone-producing glomerulosa cells, nobody had previously considered a role for anion channels in aldosterone production and hypertension."

In a separate study, FernandesRosa and Maria-Christina Zennaro also applied whole-exome sequencing to identify causal genes in 12 patients who had been diagnosed with primary aldosteronism of unknown aetiology before 25 years of age. Investigation of two index patients together with their parents and unaffected siblings to search for de novo variants led to the identification of a heterozygous p.Gly24Asp variant in CLCN2. Electrophysiological experiments in genetically modified adrenocortical cell models, performed by Thomas Jentsch and his team, as well as patch-clamp analysis of adrenal cortex glomerulosa cells in adrenal slice preparations demonstrated that the mutation leads to $\mathrm{ClC} 2$ opening, which induces depolarization of the glomerulosa cell membrane. The researchers also demonstrated that this depolarization induces opening of voltage-dependent calcium channels and that the resulting rise in cytoplasmic calcium concentration induces the expression of enzymes, such as aldosterone synthase, involved in aldosterone biosynthesis.

Both groups state that their findings suggest that CLCN2 should be included in the panel of genes that are screened in patients who develop early-onset primary aldosteronism. The findings from these studies also open new possibilities for the treatment of primary aldosteronism.

Susan J. Allison

ORIGINAL ARTICLES Scholl, U. I. et al. CLCN2 chloride channel mutations in familial hyperaldosteronism type II. Nat. Genet. https:// doi.org/10.1038/s41588-018-0048-5 (2018) | Fernandes-Rosa, F. L. et al. A gain-of-function mutation in the $C L C N 2$ chloride channel gene causes primary aldosteronism. Nat Genet. https:// doi.org/10.1038/s41588-018-0053-8 (2018) 